\title{
Efecto de un procedimiento de preparación mental en la capacidad de concentración de jugadores de un equipo de torball ${ }^{*}$
}

\section{Effect of a Mental Training Procedure on Concentration Ability of Torball Players}

\author{
Alveiro Sánchez Jiménez ${ }^{* *}$ \\ Alain Zanger*** \\ Jhon Freddy Ramírez ${ }^{* * * *}$
}

Recibido: 5 de abril de 2010

Revisado: 7 de mayo de 2010

Aceptado: 4 de junio de 2010

\section{Resumen}

La presente investigación es el resultado de una intervención en preparación mental con el equipo de Torball de Brest (Francia), fundamentalmente en las técnicas de fijación de objetivos, de la concentración y de la visualización, con el fin de reducir en competición los errores de ejecución técnica ligados a la falta de concentración

* Esta investigación es el producto final de la práctica profesional y académica durante el proceso de formación de educación superior de Alveiro Sánchez Jiménez. Estudio desarrollado con el fin de obtener el título de la Maestría en Desarrollo Personal, Recursos Mentales de las Actividades Físicas y Deportivas" de la Université de Bretagne Occidentale. Brest (Francia), durante los períodos académicos 2005-2006 y 2006-2007. UFR Sport et Education Physique (Université de Bretagne Occidentale - Brest - France). MEN EA 3883 LISyC UBO/ENIB. Centre Européen de Réalité Virtuelle (C.E.R.V.). 25 rue Claude Chappe. BP 38F 29 280. Plouzané. France.

4 Universidad Santo Tomás. Bogotá. Colombia. Correo electrónico: sanalveiro72@yahoo.com

5 Université de Bretagne Occidentale. Brest. Francia. Correo electrónico: alain.zanger@univ-brest.fr

6 Universidad Santo Tomás. Bogotá. Colombia. Correo electrónico : jhon_f_ramirez@yahoo.es 
y obtener el ascenso a la segunda división del campeonato francés de Torball.

El proceso de preparación mental ha tenido una cierta eficacia y los jugadores han mejorado su capacidad atencional, al igual que su nivel técnico, físico y motivacional.

Palabras clave: torball, preparación mental global, fijación de ob- jetivos, visualización, concentración.

\section{Abstract}

This researh present how with a global mental training procedure based on goal setting and imagery, there was a signicant decrease in the number of game mistalles by Toball players.

After two years of training and improve ment of these mental technics, the two championships january and may, have underlined an improvement on the players concentration ability. Players have made less mistakes and were more successful.

We could say that the mental training process has had a relative effectiveness. Players have made at concentration, technical, physical and motivational levels.

Key words: torball, global mental training, goal setting, mental imagery, concentration. 


\section{Introducción}

En el ámbito del deporte, un buen rendimiento frecuentemente está ligado a la capacidad de concentración en la ejecución de una acción o tarea técnica, una cualidad donde se debe tener en cuenta dos componentes: la concentración y la focalización de la atención. La primera es considerada en términos generales y por diversos autores como Schmidt, A. \& Peper, E. (1991), Weinberg, S. \& Gould, D. (1996), quienes la determinan como la habilidad de focalizar y mantener la atención sobre una tarea en curso de ejecución o sobre los estímulos relevantes del medio ambiente, y de no ser afectado y/o distraído por estímulos internos o externos no relacionados a la tarea, y la segunda, la focalización de la atención sobre las informaciones pertinentes del medio ambiente, donde se requiere ignorar o eliminar aquellas que sean inútiles.

De la Vega, M. (2003) afirma que la concentración es uno de los estados más importantes que se pueden poner al servicio del deportista siendo complemento para mejorar su rendimiento tanto en la competición como en los entrenamientos (figura 1), en donde se exponen las principales variables que se encuentran relacionadas con la concentración.

La concentración de igual forma es definida como "La focalización de toda la atención en los aspectos relevantes de una tarea, ignorando o eliminando los demás aspectos que la puedan alterar", (González. 1978 \& Samulski, 2006). En este sentido, podemos afirmar que el desarrollo de la concentración de los jugadores no debe ser ajeno a su proceso de entrenamiento, donde encontramos dos aspectos fundamentales: por un lado está la atención en las señales ambientales pertinentes, eliminando por tanto las que no lo son, y por otro está el mantenimiento de ese foco atencional.

Otros autores como Barman, 1986; Weinberg y Gould (1999); Eberspacher, H. (1995) y Thill, E. y Fleurance, P. (1998); recomiendan diferentes directrices orientadas a mejorar la capacidad de concen- 
tración en el deporte, entre las que se destacan: desarrollar entrenamientos variados para evitar la monotonía, mejorar la motivación, analizar las vivencias de éxito y/o fracaso, creación de estímulos de rendimiento, entrenamientos de simulación competitiva, utilización de palabras claves para mantener el nivel óptimo de concentración, la concentración inmediata eliminando las experiencias pasadas y la reafirmación de los pensamientos positivos.

La figura 2 relacionada con el desarrollo de la concentración en el deporte, presenta la clasificación de los aspectos propios que el individuo debe controlar a nivel fisiológico, físico, técnico, emocional y motivacional, así, la concentración se controla y se mantiene en todo proceso o en cualquier intervención deportiva, en una parte del proceso o en su totalidad.

Estos elementos son utilizados en deportes convencionales para personas sanas, pero el impacto de los mismos en deportes adaptados (torball y goalball) es poco conocido y aplicado, deportes en donde se presentan características especiales de discapacidad visual que tienen como principal rasgo el desarrollo de las cualidades auditivas, kinestésicas, táctiles, entre otras, donde se favorece la concentración, la anticipación, la memorización y el trabajo en equipo.

Es así que en el entrenamiento deportivo el proceso de preparación mental se ve beneficiado por las técnicas de concentración, elementos claves para el rendimiento en todo deporte, con una repercusión especial en Torball, donde se han sugerido para la presente investigación dos procesos específicos de intervención: una fase de observación y una fase de intervención cuyo objetivo es elevar el estado de condición física.

Ahora bien, es importante aclarar al lector, algunos conceptos importantes del estudio, entre ellos que el objetivo de la preparación mental es optimizar el nivel personal del deportista permitiéndole tener en la competición las condiciones ideales para la máxima manifestación de la condición física, Pate (1988), es decir, se trata de 


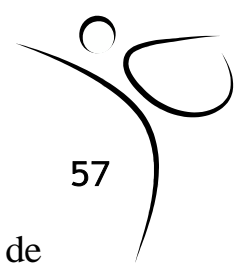

una preparación para la competición a través de un aprendizaje de aptitudes mentales (Zanger, 2007).

Sin embargo, no deben dejarse de lado las técnicas de fijación de objetivos y la de repetición mental, la primera como una técnica que guía al deportista en los aspectos más importantes para movilizar los esfuerzos con miras al objetivo final, favoreciendo la construcción de estrategias de resolución de tareas Locke, E.A. \& Latham, G.P. (2002), y la segunda, para estimular los aprendizajes y el nivel óptimo de competición Driskell, J.E., Copper, C., y Moran (1994) y Rodríguez Facal, permitiendo modificar las creencias del individuo de forma positiva en función a la interpretación que él tiene de la imaginación mental, Ahsen, A. (1984) y Murphy, S.M. (1994).

Finalmente, las técnicas de preparación mental (tabla 1), particularmente las estrategias de concentración, fijación de objetivos y de repetición mental buscan disminuir los errores de ejecución técnica relacionados con los problemas de desconcentración, lo cual lleva a plantear el presente estudio denominado efecto de un procedimiento de preparación mental en la capacidad de concentración de jugadores de un equipo de torball, que permita establecer el impacto de los elementos mencionados en una población con discapacidad visual.

\section{Metodología}

Población y muestra: La muestra del presente estudio es la totalidad de la población, ya que en la región francesa de Bretaña existen solamente dos clubes de torball, en donde uno de ellos corresponde al equipo de la ciudad de Brest. El club Handi-Brest tiene inscritos cinco jugadores que participaban en el torneo de tercera división, el cual ganó el derecho de competir en la segunda división de la temporada 2007-2008.

Como criterios de inclusión se consideraron los siguientes aspectos: haber sido practicante de Torball por un tiempo mínimo de 
tres años, con diagnóstico de discapacidad visual importante (visión inferior a 1/10), con certificación médica aportada por la Federación Francesa "Handisport" para la práctica del deporte y el aporte de su consentimiento informado. El estudio se guió atendiendo la carta de Helsinki para experimentación con humanos y fue aprobado por el comité ético de la Université de Bretagne Occidentale.

Diseño experimental y pruebas de control: este proceso metodológico se desarrolló en dos años (2005 - 2007), la primera fase (2005 2006) denominada de observación, estaba relacionada con la evaluación de la técnica y sus posibles implicaciones con la desconcentración (tiro al exterior del terreno de juego, tocar las cuerdas con el balón y/o con el cuerpo, balón perdido, falta de rapidez para regresar a posición de defensa, falta de velocidad de reacción en defensa, falta de velocidad de reacción en contra-ataque, manos sobre el suelo y mal pase), y la segunda fase (2006 - 2007), denominada de intervención, donde se aplicaron las técnicas de concentración, fijación de objetivos y visualización que inició con la administración de un cuestionario individual (figura 4) para los jugadores y el cuerpo técnico.

Los instrumentos de recolección de la información figuras 3 y 4 , fueron diseñados y avalados por la Facultad de Sciences et Technologies des Activités Physiques et Sportives STAPS de la Universidad de Bretagne Occidentale y por el club Handi-Brest. Los materiales y los métodos pertenecen a la Universidad de Bretagne Occidentale, directamente en la UFR Sport et Education Physique (Université de Bretagne Occidentale - Brest - France). MEN EA 3883 LISyC UBO/ENIB. Centre Européen de Réalité Virtuelle (C.E.R.V.). 25 Rue Claude Chappe. BP 38F 29 280. Plouzané. France.

Procedimientos: el equipo investigador empieza por determinar los posibles errores ligados a la desconcentración, los cuales han sido observados en el campeonato francés de tercera división. Luego a partir de los datos recolectados, se han creado y aplicado algunos ejercicios de "visualización" (técnica de preparación mental, donde 


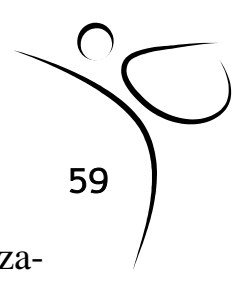

la imaginación y la repetición de acciones técnicas y de desplazamientos propios de la competición, son creados mentalmente antes de los partidos de competencia, Hall, C.R., Mack, D., Paivio, A., y Hausenblas, H.A. 1998). Aspectos asociados a una estrategia de objetivos, con el fin de mejorar las competencias de los jugadores y disminuir los momentos críticos para desarrollar un nivel óptimo de competición.

De igual forma las rutinas de entrenamiento, relacionadas con el mejoramiento del nivel técnico en ataque-defensa y la fijación de objetivos precisos, han sido construidas teniendo en cuenta los consejos prácticos de la Federación Francesa de Torball y las condiciones de eficacia de la visualización según las recomendaciones de Lang, P.J. (1979), Murphy, S. y Martin, K.A. (2002) y Henry, G. (2001).

Este proceso de intervención se desarrolla en dos momentos, el primero durante la temporada 2005 - 2006 en la fase denominada de observación, enfocada en la identificación de las acciones técnicas ligadas a la desconcentración de cada uno de los jugadores en situaciones reales de juego.

Ahora bien, finalizada esta primera fase y con la información recolectada, se inicia la segunda fase de la investigación, denominada intervención, ejecutada durante la temporada 2006 - 2007, donde se aplicaron las técnicas de concentración, fijación de objetivos y visualización. Desde esta perspectiva, se utilizó la estrategia de fijación de objetivos con el fin de conocer los intereses personales y colectivos hasta determinar el tipo de intervención más adecuado en relación con los objetivos del equipo.

Esta segunda fase inicia con la aplicación de la técnica de preparación mental, denominada goal-seting o fijación de objetivos, en donde cada jugador determinaba sus objetivos personales, deportivos y de equipo. En este sentido dicha estrategia permite guiar al deportista hacia los aspectos más importantes de la tarea a desarrollar 
para movilizar los esfuerzos con miras al objetivo final, favoreciendo la construcción de estrategias de resolución de tareas, (Locke \& Latham, 2002).

Las sesiones de entrenamiento (tabla 1) tenían una duración de dos horas y treinta minutos, dos veces por semana. Cada entrenamiento inicia con la explicación del objetivo, seguido por el calentamiento y luego la aplicación de algunos ejercicios de repetición mental para introducir a los jugadores en la técnica y la especificidad de los objetivos del equipo.

Características y puesta en escena de los ejercicios de concentración para la defensa: para el desarrollo óptimo de este proceso, se determinaron algunos elementos clave como: la correcta posición de defensa, la focalización de la atención sobre el sonido que produce el balón cuando impacta contra el suelo y luego realizar la zambullida (plongeon) para terminar nuevamente en la posición de defensa. Cada rutina tiene una duración de cinco minutos, similar al tiempo real de juego en competencia.

Para el trabajo de ataque: esta segunda secuencia de ejercicios se realiza con desplazamientos y los movimientos técnicos propios de un tiro en el ataque y fueron guiados por las normas técnicas de tiro expuestas por la federación francesa de Torball. Las indicaciones son igualmente pertinentes para la orientación y la definición de las trayectorias de tiro, es decir, la orientación táctil en los tapetes o el arco realizada con las manos o los pies a fin de identificar los costados de los tapetes para "visualizar" de manera más eficaz la portería contraria.

Técnica sobre la repetición mental: este proceso ha permitido construir rutinas de trabajo utilizadas durante las sesiones de entrenamiento y durante la competición, aplicadas justo después de terminar el proceso de calentamiento. El proceso "introduce" al jugador mentalmente en los ejercicios específicos de la técnica o de la táctica y está basado en la argumentación de la "teoría bio- 

2007) al igual los siete (7) primeros y siete (7) últimos partidos de entrenamiento del equipo durante la temporada 2005 - 2006 se presentan de la siguiente manera: balón al exterior del terreno (184 en los 25 partidos de entrenamiento) y durante la competición (23 en Mulhouse, 21 en Laval y 17 en Marseille), tocar las cuerdas con el balón (112 en los 25 partidos de entrenamiento) y durante la competición (16 en Mulhouse, 9 en Laval y 14 en Marseille), datos que de igual forma están enumerados en la tabla 2.

De otra parte, la diferencia es significativa entre estos resultados y aquellos desde el primer campeonato en Mulhouse es de $(t(6)=2.448 ; \mathrm{p}<0.005)$. De 63 errores en Mulhouse, el equipo solamente comete 45 en el campeonato de la ciudad de Laval y luego 39 en Marsella. Esta baja es significativa entre los resultados del primer campeonato en Mulhouse y del último en Marseille con un valor de $(\mathrm{t}(5)=3.335, \mathrm{p}<0.01)$.

De igual forma, es importante destacar que a partir del campeonato en la ciudad de Laval, desaparecen algunos errores de desconcentración tales como balones perdidos, tocar las cuerdas con el cuerpo o los malos pases.

\section{Discusión}

Las observaciones y el registro de los datos demuestran que han desaparecido ciertos errores durante las competiciones oficiales, tales como la rapidez para regresar a la posición de defensa o la falta de velocidad de reacción para contraatacar, donde algunos autores al estudiar la preparación mental con el equipo de Torball de Brest, como Sánchez. A. y Zanger. A. (2007), revelan algunos beneficios que puede encontrar una persona con discapacidad visual en el deporte, los cuales son incomparables en términos de autonomía, de confianza en sí mismo, de control corporal y de capacidad social, factores que generan motivaciones y responsabilidades que ayudan con el desarrollo personal del deportista con 


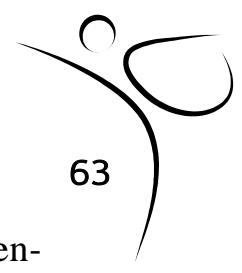

discapacidad, y que son reafirmados con los resultados del presente estudio.

Los resultados obtenidos a través de las sesiones de intervención en cada entrenamiento y durante los campeonatos, han demostrado que el mejoramiento de las capacidades de concentración son perfeccionadas durante los partidos de entrenamiento y durante la competición, cuando se atienden las recomendaciones sobre la concentración, la técnica y la táctica sugeridas en otros estudios como: desarrollar entrenamientos variados para evitar la monotonía, mejorar la motivación y analizar las vivencias de éxito y/o fracaso Samulski, D. (2006); Weinberg y Gould, (1999) y Eberspacher, H. (1995).

Entre los siete primeros partidos de entrenamiento y los siete últimos los jugadores han cometido menos errores (una baja global del $15 \%$ ) y específicamente las bajas del $21 \%, 30 \%$ y $21 \%$ respectivamente en los errores que más se han presentado durante la competencia: tiros al exterior del terreno, tocar las cuerdas con el balón y la falta de velocidad de reacción en los contraataques. Factores que gracias a la adecuada ejecución técnica se ven reflejados en la disminución de casos presentados durante la fase de competencia, así como lo sustentan investigadores para la construcción de estrategias de resolución de tareas (Locke \& Latham, 2002).

Desde el punto de vista de los investigadores y según los datos recogidos durante los partidos de entrenamiento, los jugadores ya no utilizan los mismos "recursos mentales" y atencionales que durante los campeonatos, como lo señala la tabla 2 , la cual refleja una disminución considerable en los totales de cada campeonato: 63 en Mulhouse, 45 en Laval y 39 en Marseille, y que se explica por "la focalización de toda la atención en los aspectos relevantes de una tarea, ignorando o eliminando los demás aspectos que la puedan alterar factores indispensables para el manejo de los momentos claves del juego durante la competencia" (González, 1978). 
La diferencia entre los mejores resultados de los partidos de entrenamiento (los siete últimos) y aquellos del primer campeonato antes de la intervención relacionada con la preparación mental, es significativa. $\mathrm{T}(6)=2.448 ; \mathrm{p}<0.05$, conservándose los mismos errores dominantes (tiros al exterior del terreno de juego y tocar las cuerdas con el balón) con porcentajes similares (+/- 37\% y 25\%); aspectos ligados a la técnica, los cuales se pueden explicar debido a los diferentes niveles de exigencia deportiva individual y colectiva, los cuales se ven reflejados en cada deportista en emociones y presiones psicológicas que afectan el nivel de concentración y de rendimiento de cada uno y por ende del equipo, aspectos que se han sugerido como las principales variables que se encuentran relacionadas con la concentración durante la competencia (figura 1) (De la Vega, 2003).

Igualmente, el número de errores relacionados con la desconcentración ha disminuido entre los resultados de los tres campeonatos (menos del $29 \%$ de errores), debido a que en cuatro meses de entrenamiento, ciertos errores ligados a la desconcentración han desaparecido de las fichas de observación tales como: los balones perdidos, tocar las cuerdas con el cuerpo o hacer malos pases, cambios que se podrían explicar por un aprendizaje de aptitudes mentales, las cuales generan estados de autoconfianza y memorización de los elementos técnicos propios de la fundamentación técnica del torball" (Zanger, 2007).

El equipo de Brest ha perdido un solo partido y los jugadores han marcado 34 goles, quedan aún algunos errores por corregir, ligados a la técnica de tiro y en la falta de velocidad de reacción en defensa, pero ya los jugadores cometen menos errores de ejecución técnica. Estos cambios son reforzados a través de ejercicios de visualización consistentes en la repetición mental de un gesto técnico sin mover ningún segmento corporal, dicha estrategia de preparación mental es muy utilizada en el mundo deportivo para estimular los aprendizajes y el mejoramiento de la condición física; particularmente para los deportistas con dominio cognitivo (Driskell, Copper \& Moran, 1994; Sánchez \& Zanger, 2007). 


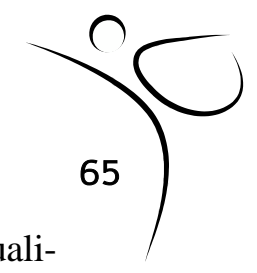

Además se deben considerar otros elementos dentro de la visualización que permitan modificar las creencias del individuo de forma positiva en función de la interpretación que él hace con la visualización, Ahsen, 1984; Murphy, 1994. Eso que puede actuar sobre el sentimiento de autoeficacia, Bandura, 1997; particularmente cuando ella está asociada con imágenes de éxitos y de competencia Moritz, Hall, Martin y Vadocz, 1996; Suinn, 1996; aspecto y técnica de preparación mental que particularmente son reafirmados por Ahsen, A. 1984; Murphy, S.M. 1994; Murphy, S. y Martin, K.A. 2002; Bandura, A. 1997; Moritz, K.J., Hall, C.R., Martin, K.A., y Vadocz, E. 1996 y Suinn, R.M. 1996.

Dichos elementos técnicos y errores de ejecución son corregidos a través de la comunicación del equipo dentro del terreno de juego; cuando se detectan los factores que influyen en el desarrollo de la concentración en el deporte, ya que es indispensable para el control emocional, fisiológico, técnico-táctico y físico que favorezcan el óptimo rendimiento deportivo durante la competición (figura 2).

Esta investigación aporta un primer estudio confiable para entrenadores y practicantes de este deporte, donde el equipo investigador hace un primer acercamiento al desarrollo de sesiones de entrenamiento específicas para el mejoramiento de las capacidades atencionales de deportistas con discapacidad visual, generando un complemento significativo al entrenamiento deportivo en torball.

El programa ha demostrado, que la aplicación de diferentes técnicas de preparación mental, en especial la concentración, la visualización y la estrategia de fijación de objetivos, son elementos claves para el adecuado desarrollo y óptimo desempeño del equipo de torball durante el campeonato francés de tercera división, logrando así el objetivo principal, el ascenso a la segunda división para la temporada 2007 - 2008.

Ahora bien, es importante recalcar que a pesar del cuidado tenido durante todo el proceso investigativo, el presente estudio tiene algunas limitaciones; pues es el primer estudio que presenta ca- 
racterísticas específicas de la preparación mental aplicadas en el Torball, las cuales hacen que esta investigación sea un primer paso para futuros estudios que permitan complementar desde diferentes perspectivas los resultados de dicha investigación.

En conclusión, cada uno de los jugadores del equipo de Brest ha sido beneficiado de un conjunto de variables para ayudarlo a estar motivado, tener confianza en sí mismo; esto se traduce en la baja de errores de ejecución ligados a la desconcentración, así mismo, al mejoramiento del nivel deportivo del equipo, aspectos que no solo se ven reflejados en el terreno de juego de torball, sino también en cada una de sus actividades cotidianas y en su inclusión a una vida social. 


\section{Anexos}

Tabla 1. La aplicación de cada una de las técnicas de preparación mental y el número de estilos por semana y por el período de intervención

\begin{tabular}{|c|c|c|c|}
\hline $\begin{array}{c}\text { Nombre de la } \\
\text { técnica }\end{array}$ & Etapas de aplicación & $\begin{array}{l}\text { Número de } \\
\text { estímulos por } \\
\text { semana }\end{array}$ & $\begin{array}{c}\text { Total de } \\
\text { sesiones } \\
\text { empleadas } \\
\text { para aplicar la } \\
\text { técnica }\end{array}$ \\
\hline $\begin{array}{l}\text { Fijación de } \\
\text { objetivos }\end{array}$ & $\begin{array}{l}\text { Esta es la primera etapa del } \\
\text { proceso de preparación men- } \\
\text { tal. En ella el objetivo principal } \\
\text { es el de conocer los objetivos } \\
\text { personales de cada jugador; en } \\
\text { aspectos como: el nivel depor- } \\
\text { tivo personal, el de los com- } \\
\text { pañeros de equipo, el trabajo } \\
\text { de los entrenadores. De igual } \\
\text { forma, conocer los objetivos } \\
\text { del jugador a nivel personal, } \\
\text { familiar y profesional. }\end{array}$ & $\begin{array}{l}1^{\text {a }} \text { Semana de } \\
\text { entrenamiento. }\end{array}$ & \begin{tabular}{l}
\multicolumn{1}{c}{1.} \\
Este proceso se \\
desarrolló en la \\
primera sesión \\
de entrenamiento \\
de la temporada \\
$2005-2006$.
\end{tabular} \\
\hline Concentración & $\begin{array}{l}\text { Esta técnica se aplica desde el } \\
\text { mismo instante de comenzar } \\
\text { el calentamiento. Luego de que } \\
\text { el entrenador fija los objetivos } \\
\text { de la sesión y los socializa con } \\
\text { el equipo; a cada jugador se le } \\
\text { solicita focalizar su atención en } \\
\text { cada uno de los objetivos pla- } \\
\text { neados para el entrenamiento. }\end{array}$ & $\begin{array}{l}\quad 2 . \\
\text { Una por cada } \\
\text { sesión de } \\
\text { entrenamiento: } \\
\text { miércoles y } \\
\text { sábados. }\end{array}$ & $\begin{array}{l}\text { Total: } \\
160 . \\
80 \text { (cuarenta) } \\
\text { sesiones de } \\
\text { trabajo. }\end{array}$ \\
\hline Visualización & $\begin{array}{l}\text { Seguido del calentamiento, } \\
\text { para cada actividad y/o ejerci- } \\
\text { cio planteado por el entrena- } \\
\text { dor, cada jugador hace la re- } \\
\text { petición mental (sin mover el } \\
\text { cuerpo) del ejercicio, siempre } \\
\text { visualizando la ejecución del } \\
\text { mismo pensando en el éxito } \\
\text { en la ejecución. }\end{array}$ & \begin{tabular}{|l|} 
\\
Una en cada sesión \\
de entrenamiento \\
miércoles y sábados.
\end{tabular} & $\begin{array}{l}\text { Total: } \\
160 . \\
80 \text { (cuarenta) } \\
\text { sesiones de } \\
\text { trabajo. }\end{array}$ \\
\hline & $\begin{array}{l}\text { Volumen total de sesiones de } \\
\text { preparación mental. }\end{array}$ & Dos por semana. & $\begin{array}{l}160 \text { sesiones. } \\
80 \text { semanas. }\end{array}$ \\
\hline
\end{tabular}


Tabla 2. Evolución de los errores de ejecución técnica ligados a un posible problema de desconcentración durante los partidos de los campeonatos de Mulhouse y Laval (2006, enero - mayo), y Marsella (2007 - enero), además de los siete primeros y siete últimos partidos de entrenamiento del equipo de Brest durante la temporada 2005 - 2006

\begin{tabular}{c|cc}
$\begin{array}{c}\text { Situaciones de } \\
\text { desconcentración }\end{array}$ & $\begin{array}{c}\text { Competición: 7 partidos por } \\
\text { campeonato }\end{array}$ & $\begin{array}{c}\text { Partidos de } \\
\text { entrenamiento }\end{array}$ \\
Mulhouse $\quad$ Laval & Marseille & 7 primeros
\end{tabular}

Tiro al exterior del terreno 23 21 17 61 48

\begin{tabular}{|l|c|c|c|c|c|}
\hline $\begin{array}{l}\text { Tocar las cuerdas con } \\
\text { el balón }\end{array}$ & 16 & 9 & 14 & 37 & 26 \\
\hline Balón perdido & 2 & 0 & 0 & 12 & 15 \\
\hline $\begin{array}{l}\text { Falta de velocidad de } \\
\text { reacción en defensa }\end{array}$ & 5 & 11 & 4 & 11 & 10 \\
\hline $\begin{array}{l}\text { Tocar las cuerdas con } \\
\text { el cuerpo }\end{array}$ & 3 & 0 & 0 & 6 & 8 \\
\hline Manos sobre el suelo & 4 & 4 & 5 & 10 & 9 \\
\hline Mal pase & 10 & 0 & 0 & 12 & 10 \\
\hline Total & 63 & 45 & 39 & 149 & 126 \\
\hline
\end{tabular}

\section{Figuras}

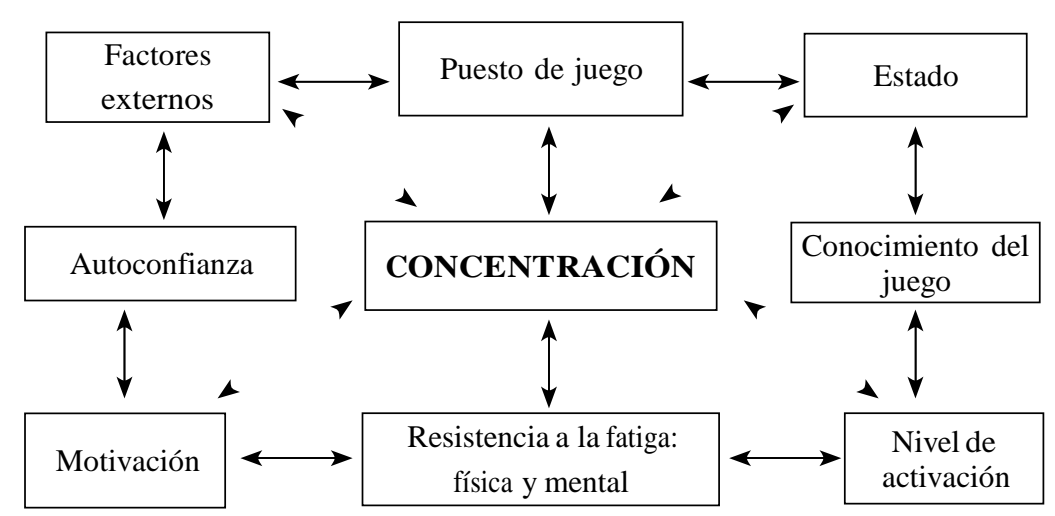

Figura 1. Principales variables que se encuentran relacionadas con la concentración Vega Marcos (2003). 


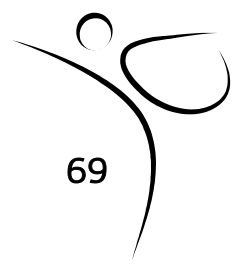

Motivación actual

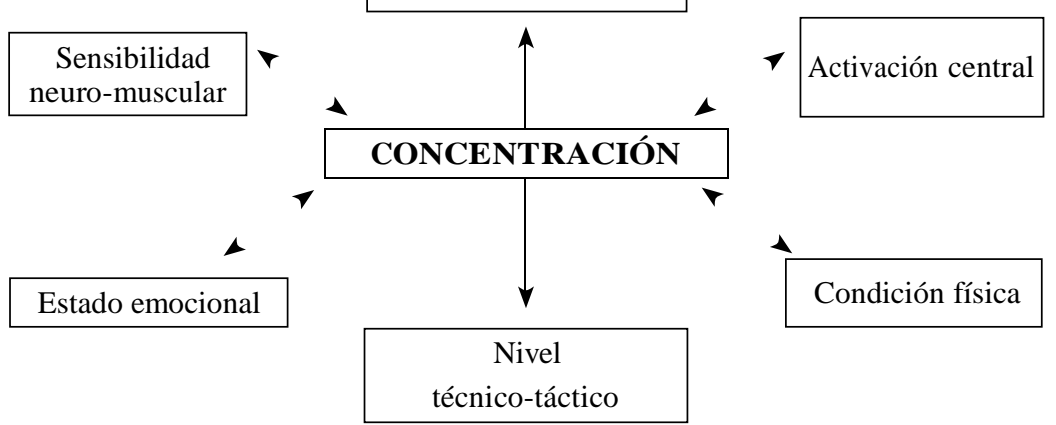

Figura 2. Desarrollo de la concentración en el deporte (Barman,1986).

UNIVERSITE DE BRETAGNE OCCIDENTALE. U.F.R. STAPS. MASTER

2. GRILLE DE RECUEIL DE DONNEES. EQUIPE DE TORBALL DE BREST. CHAMPIONNAT FRANÇAIS MARSEILLE 2007.

Brest Vs :

\begin{tabular}{|c|c|c|c|c|c|c|c|c|}
\hline \multicolumn{2}{|c|}{ JOUEUR } & \multicolumn{6}{|c|}{ Premier mini temps. } & Deuxième mini temps. \\
\hline \multicolumn{2}{|c|}{ Jean Pierre } & & & & & & & \\
\hline Stress AV & 1 & 2 & 3 & 4 & 5 & 6 & 7 & \\
\hline Stress AP & 1 & 2 & 3 & 4 & 5 & 6 & 7 & \\
\hline Fatigue AV & 1 & 2 & 3 & 4 & 5 & 6 & 7 & \\
\hline Fatigue AP & 1 & 2 & 3 & 4 & 5 & 6 & 7 & \\
\hline \multicolumn{2}{|l|}{ Steven } & & & & & & & \\
\hline Stress AV & 1 & 2 & 3 & 4 & 5 & 6 & 7 & \\
\hline Stress AP & 1 & 2 & 3 & 4 & 5 & 6 & 7 & \\
\hline Fatigue AV & $\mathbf{1}$ & 2 & 3 & 4 & 5 & 6 & 7 & \\
\hline Fatigue AP & 1 & 2 & 3 & 4 & 5 & 6 & 7 & \\
\hline Arnaud & & & & & & & & \\
\hline
\end{tabular}




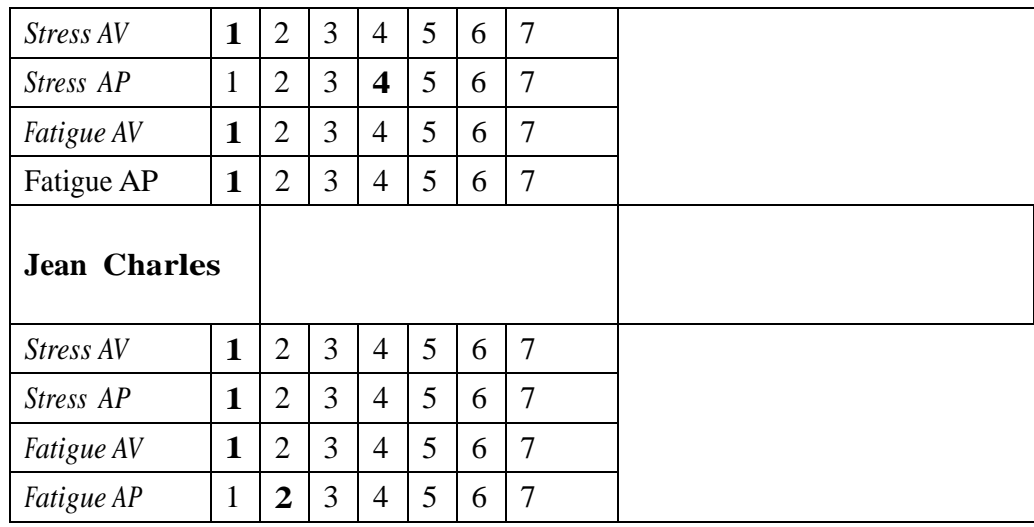

Figura 3. Planilla de registro de datos, equipo de Torball de Brest. Campeonato francés de tercera división, 2007.

UNIVERSITE DE BRETAGNE OCCIDENTALE. U.F.R. SPORT ET EDUCATION PHYSIQUE. MASTER 2. SPORT, SANTE, SOCIETE. Stage Professionnel.

Questionnaire aux joueurs de l'équipe de Torball de Brest.

Bonjour $\mathrm{Mr}$

le but de ce questionnaire est de connaître vos objectifs personnels, sportifs et professionnels PAR RAPPORT ì VOTRE PRATIQUE DE TORBALL ; pendant la saison 2006-2007.

Je vous remercie de donner l'information la plus claire et précise*.

1. Quels sont vos objectifs par rapport à votre vie personnelle? Ex:Travailler en équipe.

a.

b.

c.

d.

e. 


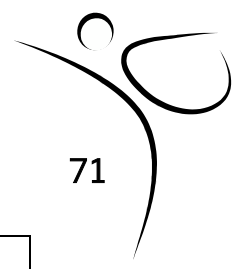

2. Quels sont vos objectifs par rapport à votre pratique de Torball? Ex:

Améliorer ma vitesse de réaction en défense.

a.

b.

c.

d.

e.

3. Quels sont vos objectifs par rapport à l'équipe de Torball de Brest? Ex : Avoir une communication positive avec mes partenaires.

a.

b.

c.

d.

e.

4. Quels sont vos objectifs par rapport à vos partenaires, le coach et l'entraîneur $* *$ ?

Jean Pierre :

Arnaud :

Jean Charles :

Yannick :

Steven :

Jeff :

Alveiro :

*si vous avez besoin d'écrire de plus des objectifs, vous pouvez utiliser une autre feuille.

**Dans votre nom, écrivez voue une $\mathrm{X}$.

Figura 4. Cuestionario de objetivos, 2007. 


\section{Referencias}

Ahsen, A. (1984). ISM: The Triple Code Model for Imagery and Psychophysiology. Journal of Mental Imagery, 8 .

Bandura, A. (1997). Self-efficay: The Exercise of Control. New York: Freeman.

Barman (1986). Desarrollo de la concentración en el deporte. Manual para educación física, psicología y fisioterapia. En: Revista Kinesis, Vol. 1-2.

De la Vega, M. (2003). Importancia de la concentración en el fútbol base: una perspectiva aplicada. En: Cuadernos de psicología del deporte,Vol. 3, No. 2. Murcia: Facultad de Psicología de la Universidad de Murcia.

Driskell, J.E.; Copper, C., \& Moran (1994). Does Mental Practice Enhance Performance? Journal of Applied Psychology, No. 79.

Eberspacher, H. (1995). Entrenamiento mental. Zaragoza: Inde Publicaciones.

González. M.(1978). Concentración psicológica. Ediciones Mensajero.

Hall, C.R., et ál. (1998). Imagery Use by Athletes: Development of the Sport Imagery Questionnaire. International Journal of Sport Psychology, 29.

Henry, G. (2001). An Individualized Feedback System for tennis players, A case study, Journal of Sport Psychology.

Lang, P.J.(1979). A Bio-informational Theory of Emotional Imagery. Psychophysiology, 16. 
Locke, E.A. \& Latham, G.P. (2002). Building a Practically Useful Theory of Goal Setting and Task Motivation. In: American Psychologist, 57.

Moritz, K.J., et ál. (1996). What are Confident Athletes Imaging? An Examination of Image Content. The Sport Psychologist.

Murphy, S.M. (1994). Imagery Interventions in Sport. Medicine and Science in Sports and Exercise, 26, 486-494.

Murphy, S. \& Martin, K.A. (2002). The Use of Imagery in Sport, in T. Horn (Ed), Advances in Sport Psychology, (2ed, pp.405-439). Champaign, IL: Human Kinetics.

Pate (1988). Condición física relacionada con la salud en escolares de 10 años de edad de Granada.

Rodríguez, F. (1995) Entrenamiento de la capacidad de salto. Santa Fe Books, El entrenamiento pliométrico.

Samulski, D. (2006). Psicología del deporte. Manual para educación física, psicología y fisioterapia. En: Revista Kinesis, Vol. 1-2.

Sánchez. A. \& Zanger. A. (2007). La Preparation Metal de l'équipe de Torball de Brest. Trabajo de Grado. Universidad de Bretagne Occidentale.

Schmidt,A., \& Peper, E. (1991).Training Strategies for Concentration, in Williams (J.M.) (Ed.), Applied Sport Psychology: Personal Growth to Peak Performance. Mountain View, CA, Mayfield Publishing Company, 2e édition.

Suinn, R.M. (1996). Imagery Rehearsal: A Tool for Clinical Practice. Psychotherapy in Private Practice, 15. 
Thill, E. \& Fleurance, P.(1998). Guide pratique de la préparation psychologique du sportif. Paris:Vigot.

Weinberg, S. \& Gould, D. (1996). Fundamentos de Psicología del Deporte y el Ejercicio Físico. Barcelona: Ariel Psicología.

Weinberg \& Gould, (1999). Fundamentos de psicología del deporte y el ejercicio físico. Barcelona: Ariel Psicología.

Zanger,A. (2007). La preparación mental del equipo de Torball de Brest. Trabajo de Grado. Universidad de Bretagne Occidentale. 
$\sigma^{2}$<smiles>CCC1CC(CC)C(CC)O1</smiles> 